\title{
Mesoscopic simulations of the counterion-induced electro-osmotic flow: A comparative study
}

\author{
Jens Smiatek, ${ }^{1, \text { a) }}$ Marcello Sega, ${ }^{2, b)}$ Christian Holm, ${ }^{2,3, c)}$ Ulf D. Schiller, ${ }^{3, d)}$ and \\ Friederike Schmid ${ }^{1,4}$ \\ ${ }^{1}$ Physics Faculty, Universität Bielefeld, D-33615 Bielefeld, Germany \\ ${ }^{2}$ Frankfurt Institute for Advanced Studies, D-60438 Frankfurt, Germany \\ ${ }^{3}$ Max-Planck-Institute for Polymer Research, D-55128 Mainz, Germany \\ ${ }^{4}$ Institut für Physik, Universität Mainz, D-55099 Mainz, Germany
}

(Received 22 January 2009; accepted 20 May 2009; published online 22 June 2009)

\begin{abstract}
We present mesoscopic simulations of the counterion-induced electro-osmotic flow in different electrostatic coupling regimes. Two simulation methods are compared, dissipative particle dynamics (DPD) and coupled lattice-Boltzmann/molecular dynamics (LB/MD). A general mapping scheme to match DPD to LB/MD is developed. For the weak coupling regime, analytic expressions for the flow profiles in the presence of partial-slip as well as no-slip boundary conditions are derived from the Poisson-Boltzmann and Stokes equations, which are in good agreement with the numerical results. The influence of electrofriction and partial slip on the flow profiles is discussed.
\end{abstract}

(c) 2009 American Institute of Physics. [DOI: 10.1063/1.3152844]

\section{INTRODUCTION}

Microfluidic devices such as biomicroelectronicalmechanical systems and nanoelectronical-mechanical systems have attracted broad interest over the last years due to their huge potential in biotechnology. ${ }^{1,2}$ The flow profiles in such micro- or nanosized devices are strongly influenced by the properties of the boundaries due to the large surface-tovolume ratio in these systems. Surface characteristics such as the wetting behavior and/or slippage have a dramatic effect on the microscopic flow, leading to sometimes unexpected behavior. $^{3}$

One particularly important mechanism is electro-osmotic transport: In contact with a liquid, many materials commonly used in nanotechnology (e.g., polydimethylsiloxane) become charged due to ionizations of surface groups. ${ }^{4}$ As a consequence, surfaces are often covered by a compensating counterion layer. ${ }^{5}$ In an external electric field, the ions are driven in one direction, dragging the surrounding solvent with them. As a result, a flow is induced in the fluid, the electro-osmotic flow (EOF). This electrokinetic effect has numerous consequences. For example, it alters drastically the migration dynamics of mesoscopic objects such as polyelectrolytes or colloids. ${ }^{6}$ In microchannels, the EOF generated at the channel walls results in a total net flow, which is technologically

\footnotetext{
${ }^{a)}$ Present address: Institute of Physical Chemistry, University of Münster, Corrensstraße 28/30, D-48149 Münster, Germany. Electronic mail: smiatek@physik.uni-bielefeld.de.

${ }^{b)}$ Present address: Department of Physics, University of Trento, and INFN, via Sommarive 14, 38100 Trento, Italy.

${ }^{c}$ Present address: Institut für Computerphysik, Universität Stuttgart, Pfaffenwaldring 27, 70569 Stuttgart, Germany.

d)Present address: Chemical Engineering Department, University of Florida, Gainesville, FL 32611-6005, USA. On leave from Institut für Festkörperforschung, Forschungszentrum Jülich, D-52425 Jülich, Germany.
}

attractive because it can be controlled and manipulated more easily on the submicrometer scale than pressure- or sheardriven flow.

As analytical predictions for flow in such complex systems are often hard to derive, ${ }^{5}$ numerical simulations are used to investigate it in detail. The requirements on computer simulations are high: They must include the full hydrodynamics of the fluid while guaranteeing, at the same time, optimal computational efficiency. This has led to the development of a number of coarse-grained mesoscopic simulation schemes. Several methods such as lattice gas automata, ${ }^{7}$ the lattice-Boltzmann (LB) method, ${ }^{8-10}$ dissipative particle dynamics (DPD), ${ }^{11-13}$ and multiparticle collision ${ }^{14,15}$ dynamics are nowadays used to model liquids at a coarse-grained level. A completely discretized approach to study EOF via a generalized LB method has been proposed by Warren, ${ }^{16}$ and later extended by Capuani et al., ${ }^{17}$ relying on a solution of the electrokinetic equations on the LB lattice.

Compared to atomistic molecular dynamics (MD) simulations, these approaches give access to much longer time and length scales ${ }^{18}$ and are therefore suited to study the longtime behavior of soft matter systems and transport phenomena. Although the theoretical background of these methods is well understood, their lattice/off-lattice and thermal/athermal characters impede a straightforward mapping between them. Comparative studies of specific soft matter problems with different simulation methods are therefore scarce. In this paper we present the results of DPD- and coupled LB/MD simulations for the counterion-induced EOF without salt ions in a slit pore. Although this geometry does not allow to test the full coupling between ions and solvent, it has the advantage of possessing an analytical solution, which allows a precise test of the accuracy of the methods. A systematic approach to match DPD and LB/MD simulations is presented.

As mentioned earlier, flow profiles depend heavily on the boundary conditions at the surfaces. ${ }^{3}$ We derive an ana- 
lytic equation for the counterion-induced EOF in the presence of no-slip as well as partial-slip boundaries for the regime of "weak electrostatic coupling," the regime where the Poisson-Boltzmann theory is valid, and compare it to our simulation results. Furthermore, we also study the influence of the discrete character of wall charges, compared to perfectly homogeneous walls.

The paper is organized as follows. In Sec. I we derive the analytical solution for the flow profile of the counterioninduced EOF in the presence of arbitrary slip conditions. The two mesoscopic simulation methods are introduced and the general mapping scheme is discussed in Sec. III. Section IV gives the details of the simulations, and Sec. V focuses on the numerical results. We conclude with a brief summary.

\section{THEORETICAL BACKGROUND}

When brought in contact with a liquid, most materials acquire charges either by the ionization or dissociation of surface groups or the adsorption of ions from solution. In microchannels, the walls are thus typically charged and attract a layer of compensating counterions. In the following, we consider the simple situation of fluid flow in a slit channel with charged walls and dissolved counterions. Additional charges (e.g., salt ions) are not present. Depending on the relative strength of Coulomb interactions in the system, one distinguishes between several electrostatic coupling regimes, which can be realized by changing the surface charge densities, by using counterions of different valencies, or by varying the temperature. One parameter that discriminates between regimes is the electrostatic coupling constant,

$$
\Xi=2 \pi Z^{3} \lambda_{B}^{2} \sigma_{A},
$$

which gives the strength of electrostatic interactions between the counterion and the surface compared to the thermal energy. The parameters entering $\Xi$ are the Bjerrum length $\lambda_{B}$ $=e^{2} / 4 \pi \epsilon_{r} k_{B} T$, the unit charge $e$, the counterion valency $Z$, the thermal energy $k_{B} T$, the dielectric constant $\epsilon_{r}$, and the surface charge density $\sigma_{A} \cdot{ }^{19-21}$ Low values of $\Xi$ correspond to the "weak coupling limit," where the counterions at the wall are distributed in a broad diffusive layer which is well described by the Poisson-Boltzmann theory. ${ }^{22,23}$ This regime is realized at moderate surface charge densities and for monovalent ions. High values of $\Xi$ are obtained for high surface charge densities, low temperatures, or multivalent charges, and lead to the formation of nearly flat, highly adsorbed and massively correlated counterion layers. ${ }^{24}$ If in addition the two counterion layers are well separated, i.e., the slit width $d$ is much larger than the lateral distance between ions $a[d / a>1$ (Ref. 20)], one enters the "strong coupling limit," which in terms of the electrostatic coupling constant is given for $\Xi>(d / \mu)^{2}$ with the Gouy-Chapman length $\mu$ $=\left(2 \pi Z \lambda_{B} \sigma_{A}\right)^{-1}$.

In the following we focus on the analytical derivation of the EOF profiles in the weak coupling regime. The electrostatic potential $\psi(z)$ and the ion density distribution $\rho(z)$ can be calculated from the Poisson-Boltzmann equation, ${ }^{22,23}$ which reads in our case (slit channel, no salt, counterions only)

$$
\frac{\partial^{2}}{\partial z^{2}} \psi(z)=-\frac{Z e}{\epsilon_{r}} \rho(z)=-\frac{Z e}{\epsilon_{r}} \rho_{0} e^{-Z e \psi(z) / k_{B} T},
$$

where $Z e$ is the charge of the ions and $\rho_{0}$ the reference ion density at $\psi=0$. If an additional electric field $E_{x}$ is applied, the counterions move in the direction of the field and drag the solvent particles along. A net flow is induced, the counterion-induced EOF. The actual flow profile in the limit of low Reynolds numbers can be calculated with the Stokes equation, which reads in the absence of pressure gradients

$$
\eta \frac{\partial^{2}}{\partial z^{2}} v_{x}(z)=Z e \rho(z) E_{x},
$$

with the dynamic viscosity $\eta$. We impose the most general hydrodynamic boundary condition, the partial-slip condition,

$$
\left.\partial_{z} v_{x}(z)\right|_{z= \pm z_{B}}=\mp \frac{1}{\delta_{B}} v_{x}\left( \pm z_{B}\right),
$$

at the hydrodynamic wall boundaries $\pm_{B}$ with the slip length $\delta_{B}$. Comparing Eq. (3) with Eq. (2), we obtain by straightforward integration of Eq. (3) with Eq. (4) the general relation

$$
v_{x}(z)=\frac{\epsilon_{r} E_{x}}{\eta}\left(\psi\left(z_{B}\right)-\psi(z)-\delta_{B} \psi^{\prime}\left(z_{B}\right)\right) .
$$

Specifically, the solution of Eq. (2) in the slit geometry is given by ${ }^{4}$

$$
\psi(z)=\frac{k_{B} T}{Z e} \log \left(\cos ^{2}(\kappa z)\right),
$$

with the screening constant $\kappa^{2}=(Z e)^{2} \rho_{0} / 2 \epsilon_{r} k_{B} T$, where $\rho_{0}$ is the counterion density in the middle of the channel. The counterion density distribution is then given by

$$
\rho(z)=\frac{\rho_{0}}{\cos ^{2}(\kappa z)},
$$

and the electric field is

$$
E(z)=-\frac{\partial}{\partial z} \psi(z)=-\frac{2 \kappa k_{B} T}{Z e} \tan (\kappa z) .
$$

For the flow profile, we finally get

$$
\begin{aligned}
v_{x}(z)= & \frac{e}{4 \pi \lambda_{B} Z \eta} E_{x}\left(\log \left(\cos ^{2}\left(\kappa z_{B}\right)\right)-\log \left(\cos ^{2}(\kappa z)\right)\right. \\
& \left.+2 \kappa \delta_{B} \tan \left(\kappa z_{B}\right)\right),
\end{aligned}
$$

where we have expressed $\epsilon_{r}$ in terms of the Bjerrum length $\lambda_{B}$.

If the surface charges are high, the ions have high valency, or the temperature is low, charge correlations and charge fluctuations become important and mean-field approaches such as the Poisson-Boltzmann approach fail. In this case, asymptotic analytical expressions for the ion distribution in the channel can be obtained with field-theoretic methods. ${ }^{19-21}$ If the plate distance $d$ is much larger than the Gouy-Chapman length, the counterion density distribution between two planar highly charged surfaces is given by ${ }^{20}$ 


$$
\rho(z)=\frac{2 \pi \lambda_{B} \sigma_{A}^{2}}{\left(1-e^{-d / \mu}\right)}\left(e^{-(z+d / 2) / \mu}+e^{-(d / 2-z) / \mu}\right) .
$$

This result characterizes the strong coupling limit, corresponding to two independent highly self-correlated counterion layers.

\section{SIMULATION METHODS}

\section{A. DPD}

DPD is a coarse-grained particle-based simulation method for fluid dynamics. It is Galilean invariant, generates a well-defined canonical ensemble at equilibrium, and guarantees the conservation of momentum. The system consists of a set of particles with continuous positions $\mathbf{r}_{i}$ and velocities $\mathbf{v}_{i}$ whose time evolution is described by Newton's equations of motion. The forces on one particle are given by

$$
\mathbf{F}_{i}^{\mathrm{DPD}}=\sum_{i \neq j}\left(\mathbf{F}_{i j}^{C}+\mathbf{F}_{i j}^{D}+\mathbf{F}_{i j}^{R}\right),
$$

with the conservative force $\mathbf{F}_{i j}^{C}$, a two-particle dissipative interaction,

$$
\mathbf{F}_{i j}^{D}=-\gamma \omega\left(r_{i j}\right)\left(\hat{r}_{i j} \cdot \mathbf{v}_{i j}\right) \hat{r}_{i j},
$$

with $\hat{r}_{i j}=\mathbf{r}_{i j} / r_{i j}, \mathbf{r}_{i j}=\mathbf{r}_{j}-\mathbf{r}_{i}$, and a corresponding stochastic force $\mathbf{F}_{i j}^{R}$,

$$
\mathbf{F}_{i j}^{R}=\sqrt{2 \gamma k_{B} T \omega\left(r_{i j}\right)} \hat{\zeta}_{i j} \hat{r}_{i j} .
$$

The weight function $\omega(r)$ can be chosen at will-here we use the most common form $\omega(r)=1-r / r_{c}$ for $r<r_{c}[\omega(r)=0$ otherwise], with the DPD cutoff radius $r_{c}$. The random numbers $\hat{\zeta}_{i j}$ are symmetric and otherwise uncorrelated $\left(\hat{\zeta}_{i j}=\hat{\zeta}_{j i}\right)$ with mean zero and variance one. The symmetry properties $F_{i j}^{D}=-F_{j i}^{D}$ and $F_{i j}^{R}=-F_{j i}^{R}$ ensure that the total momentum is conserved in the absence of spatially varying external (conservative) potentials.

The hydrodynamic boundary condition at the walls (4) is realized with a recently developed method ${ }^{25}$ that allows to implement arbitrary partial-slip boundary conditions: We introduce an additional coordinate-dependent viscous force that mimicks the wall/fluid friction,

$$
\mathbf{F}_{i}^{L}=\mathbf{F}_{i}^{D}+\mathbf{F}_{i}^{R},
$$

with a dissipative contribution,

$$
\mathbf{F}_{i}^{D}=-\gamma_{L} \omega_{L}(z)\left(\mathbf{v}_{i}-\mathbf{v}_{\text {wall }}\right),
$$

coupling to the relative velocity $\left(\mathbf{v}_{i}-\mathbf{v}_{\text {wall }}\right)$ of the particle with respect to the wall and a stochastic force,

$$
F_{i, \alpha}^{R}=\sqrt{2 \gamma_{L} k_{B} T \omega_{L}(z)} \chi_{i, \alpha},
$$

which satisfies the fluctuation-dissipation relation and thus ensures the Boltzmann distribution to be the local equilibrium distribution. Here $\alpha$ is $x, y, z$ and $\chi_{i, \alpha}$ is a Gaussian distributed random variable with mean zero and variance one: $\left\langle\chi_{i, \alpha}\right\rangle=0,\left\langle\chi_{i, \alpha} \chi_{j, \beta}\right\rangle=\delta_{i j} \delta_{\alpha \beta}$. The viscous coupling between fluid and wall is realized by the locally varying viscosity $\gamma_{L} \omega_{L}(z)$ with $\omega_{L}(z)=1-z / z_{c}$ up to a cutoff distance $z_{c}$. Beyond $z \leq z_{c}$, the fluid satisfies the Stokes equation with an effective boundary condition of the form (4) ${ }^{25}$ The prefactor $\gamma_{L}$ can be used to tune the strength of the friction force and hence the value of the slip length $\delta_{B}$. We note that $\delta_{B}$ is an effective parameter and not related to the actual slip at the physical walls, which is usually nonzero even at $\delta_{B}=0$. Within this approach it is possible to tune the hydrodynamic boundary condition systematically from full slip to no slip and to derive an analytic expression for the slip length as a function of the model parameters. ${ }^{25}$ Theorists tend to favor no-slip boundary conditions. In microchannels, however, partial-slip boundary conditions are also observed. ${ }^{26-28}$ Here we will show results for both no-slip and partial-slip walls.

\section{B. LB approach}

In contrast to DPD, the LB method can be seen as a discrete formulation of the Boltzmann equation on a lattice, which, by means of a Chapman-Enskog expansion leads to the Navier-Stokes equation in the incompressible limit. ${ }^{29,30}$ The basic evolution equation for the mass density $n_{i}(\mathbf{r}, t)$ assigned to a discrete velocity $\mathbf{c}_{i}=\hat{\mathbf{c}}_{i} a / \tau$ on a lattice node $\mathbf{r}$ at time $t$ is given by

$$
n_{i}\left(\mathbf{r}+\mathbf{c}_{i} \tau, t+\tau\right)=n_{i}(\mathbf{r}, t)+\sum_{j} L_{i j}\left(n_{j}(\mathbf{r}, t)-n_{j}^{\mathrm{eq}}(\rho, \mathbf{u})\right),
$$

where $\tau$ is the time step and $\hat{\mathbf{c}}_{i}$ is a set of vectors connecting the grid point $\mathbf{r}$ to its nearest and next-nearest neighbors on a simple cubic lattice with lattice spacing $a$. The zero-velocity vector is also included (D3Q19 model). The collision matrix $L$ relaxes the $n_{i}$ toward the local pseudoequilibrium $n_{i}^{\mathrm{eq}}(\rho, \mathbf{u})$, which depends on the local mass density $\rho(\mathbf{r}, t)=\sum_{i} n_{i}(\mathbf{r}, t)$ and the local fluid velocity $\mathbf{u}(\mathbf{r}, t)=(1 / \rho) \sum_{i} n_{i}(\mathbf{r}, t) \mathbf{c}_{i}$. The functional form of the pseudoequilibrium is taken as the second-order approximation,

$$
n_{i}^{\mathrm{eq}}(\rho, \mathbf{u})=\rho a^{c_{i}}\left(1+\frac{\mathbf{u} \cdot \mathbf{c}_{i}}{c_{s}^{2}}+\frac{\left(\mathbf{u} \cdot \mathbf{c}_{i}\right)^{2}}{2 c_{s}^{4}}-\frac{u^{2}}{2 c_{s}^{2}}\right),
$$

which maximizes the entropy of the underlying generalized lattice gas model. ${ }^{30} c_{s}$ is the speed of sound and the parameters $a^{c_{i}}$ are weight factors that depend on the magnitude of the velocity vectors $\hat{\mathbf{c}}_{i}$ but not their direction. For the D3Q19 model the respective values are $a^{0}=1 / 3, a^{1}=1 / 18$, and $a^{\sqrt{2}}$ $=1 / 36$ and the speed of sound is $c_{s}=\sqrt{1 / 3} a / \tau$.

In order to reproduce Brownian motion in a suspension, thermal fluctuations have to be introduced. The fluctuating LB equation is given by

$$
\begin{aligned}
n_{i}\left(\mathbf{r}+\mathbf{c}_{i} \tau, t+\tau\right)= & n_{i}(\mathbf{r}, t)+\sum_{j} L_{i j}\left(n_{j}(\mathbf{r}, t)-n_{j}^{\mathrm{eq}}(\rho, \mathbf{u})\right) \\
& +n_{i}^{\prime}(\mathbf{r}, t) .
\end{aligned}
$$

For details regarding the implementation of the stochastic term $n_{i}^{\prime}(\mathbf{r}, t)$ we refer to Ref. 30 .

A delicate task in lattice-based simulations is the correct coupling between the discrete nodes of the LB-solvent and the continuous positions of the (ion) particles. This can be achieved with a Stokes-type friction force, ${ }^{31}$ 


$$
\mathbf{F}_{m l}=-\zeta[\mathbf{V}-\mathbf{u}(\mathbf{R}, t)]+\mathbf{f},
$$

which is exerted on a solute particle moving through the surrounding viscous fluid. The random force $\mathbf{f}$ is required to guarantee thermal equilibrium of the coupled system, and its amplitude can be obtained from the fluctuation dissipation relation $\left\langle f_{\alpha}(t) f_{\beta}\left(t^{\prime}\right)\right\rangle=2 \zeta k_{B} T \delta_{\alpha \beta} \delta\left(t-t^{\prime}\right)$. Momentum is exchanged between the particles and the LB fluid according to total momentum conservation in the system. The LB simulations were carried out for no-slip boundary conditions only, which were realized by applying bounce-back boundary conditions. ${ }^{32}$

\section{Parameter mapping}

Our scheme for mapping the parameters of the different simulation methods onto each other is based on the requirement that the hydrodynamic flow phenomena should be the same. Therefore, the values of the parameters entering the Stokes equation (3) should be identical, i.e., the solvent density $\rho$ and the dynamic viscosity $\eta$. Matching the solvent density $\rho$ is trivial because it is an input parameter both in the DPD and the LB method. Matching the dynamic viscosity $\eta$ is more difficult. This parameter is an input parameter in the LB method, but an a priori unknown intrinsic fluid property in DPD models. A mean-field analysis of the DPD method $^{33}$ shows that it mainly depends on the friction coefficient $\gamma$, the fluid density $\rho$, the temperature $T$, and the cutoff range of DPD interactions $r_{c}$. For given $\rho$ and $T$, it can hence be adjusted by varying $\gamma$ and/or $r_{c}$. The dynamic viscosity can be determined either by measuring the autocorrelation function of the pressure tensor in free solution, which is related to the viscosity via a Green-Kubo equation, ${ }^{34}$ or by simulating plane Poiseuille flow in a confined microgeometry, ${ }^{25}$ respectively, in free periodic boxes. ${ }^{35}$ The resulting flow profile is then fitted to a parabola $v_{x}(z)=\alpha\left(z_{p}^{2}\right.$ $-z^{2}$ ) with the magnitude

$$
\eta=\frac{\rho F_{x}}{2 \alpha}
$$

where $F_{x}$ is the body force on the solvent which was applied to generate the Poiseuille flow. Having fixed $\rho$ and $\eta$, a third parameter remains which is related to the long-time dynamical behavior of single particles, i.e., to the self-diffusion. This is another intrinsic fluid property in DPD fluids. The LB method does not operate with particles originally, but in the hybrid LB/MD-method (see Sec. III B), particles can be introduced which are coupled to the LB nodes with a coupling constant $\zeta$ [cf. Eq. (20)]. Therefore the self-diffusion coefficient $D$ can be tuned by varying the coupling constant $\zeta$ until it is in agreement with the self-diffusion coefficient of a solute particle in the DPD simulations. This can be checked by comparing the velocity autocorrelation function of a particle in both systems which is connected to the self-diffusion coefficient $D$ by a Green-Kubo expression or by simple comparison of the mean-square displacement of a tracer particle. ${ }^{34}$
TABLE I. Charge $q$, surface ion density $\sigma_{s}$, Gouy-Chapman length $\mu$, and coupling constant $\Xi$ for different electrostatic coupling regimes.

\begin{tabular}{lclll}
\hline \hline Regime & $q\left[\sqrt{k_{B} T \lambda_{B}}\right]$ & $\sigma_{s}\left[1 / \sigma^{2}\right]$ & $\mu[\sigma]$ & $\Xi$ \\
\hline Weak coupling & 1 & 0.2083 & 0.764 & 1.31 \\
Intermediate coupling & 2 & 0.042 & 0.955 & 4.19 \\
\hline
\end{tabular}

\section{SIMULATION DETAILS}

All simulations have been carried out with extensions of the freely available software package ESPRESSO. ${ }^{36-38}$ We use a cubic simulation box $(12 \sigma \times 12 \sigma \times 12 \sigma)$ which is periodic in the $x$ - and $y$-directions and confined by impermeable walls in the $z$ direction. Electrostatics for homogeneously charged walls are calculated by P3M (Ref. 39) and the ELC algorithm (Electrostatic layer correction) $)^{40}$ for $2 D+h$ slab geometries with an ELC gap size of $2 \sigma$. See Ref. 41 for a definition of the parameters. In addition, the MMM2D algorithm ${ }^{42,43}$ was used to study homogeneously charged walls in the same geometry. Ions have a mass $m$ and carry a charge $q$. They interact via a Weeks-Chandler-Andersen (WCA) potential ${ }^{44} V_{i j}=4 \epsilon\left((\sigma / r)^{12}-(\sigma / r)^{6}\right)+\epsilon$ with energy parameter $\epsilon$ and cutoff distance $r_{\mathrm{WCA}}=2^{1 / 6} \sigma$, and a Coulomb potential with Bjerrum length $\lambda_{B}=1.0 \sigma$. The parameters $\epsilon, \sigma$, and $m$ shall be used as the natural units of our system hereafter. To study the counterion-induced EOF, the walls were charged by placing discrete charges $q$ randomly all over them. The charge configuration in each wall was identical for both methods with a surface ion density $\sigma_{s}$ (the surface charge density is then given by $\sigma_{A}=q \sigma_{s}$ ). The number of counterions in the fluid matches the number of charges in the wall, such that the system is overall neutral in the case of inhomogeneously charged walls. The case of homogeneously charged walls can be realized simply by having both walls uncharged. This is due to the fact that the electric fields generated by the two plates cancel each other exactly within the slit, and the charge density profile is generated only by the internal Coulomb interaction between counterions.

Different coupling regimes were obtained by varying the ion charge $q$, the surface ion density $\sigma_{s}$, and the corresponding counterion number from 12 to 60 . The parameters are shown in Table I together with the resulting coupling constant $\Xi$. The density of solvent particles was chosen $\rho_{l}$ $=3.75 \sigma^{-3}$ and the temperature $k_{B} T=1 \epsilon$ in all simulations.

\section{A. DPD simulations}

In the DPD simulations, the walls are located at $z_{\text {wall }}$ $=(0,10) \sigma$. They interact with fluid particles and ions via a WCA potential ${ }^{44}$ with the same parameters as above $\left(\epsilon_{\text {wall }}\right.$ $\left.=\epsilon, \sigma_{\text {wall }}=\sigma\right)$. The solvent particles have the same mass as the ions $(m)$, but do not interact with other particles except the walls. The DPD friction coefficient was chosen $\gamma$ $=5.0 \sigma^{-1}(m \epsilon)^{1 / 2}$ and the cutoff range of the DPD interactions $r_{c}=1.0 \sigma$. Tunable slip boundary conditions ${ }^{25}$ were used with friction coefficients $\gamma_{L}=0.96,1.4049,3.1 \sigma^{-1}(m \epsilon)^{1 / 2}$, and $\gamma_{L}$ $=6.1 \sigma^{-1}(m \epsilon)^{1 / 2}$ with the friction range $z_{c}=2.0 \sigma$ starting at $z_{\text {wall }}=(0,10) \sigma$. The DPD timestep is $\delta t=0.01 \sigma(m / \epsilon)^{1 / 2}$. 
TABLE II. Time needed for computing a single integration step in the DPD method with tunable-slip boundary conditions (DPD+TSC) and electrostatics $(\mathrm{DPD}+\mathrm{TSC}+\mathrm{CS})$ in comparison to the LB method with bounce-back boundary conditions $(\mathrm{LB}+\mathrm{BBC})$ and electrostatics $(\mathrm{LB}+\mathrm{BBC}+\mathrm{CS})$.

\begin{tabular}{lcccc}
\hline \hline Methods & DPD+TSC & LB+BBC & DPD+TSC+CS & LB+BBC+CS \\
\hline Time/step (s) & 0.09 & 0.01 & 0.22 & 0.14 \\
\hline \hline
\end{tabular}

\section{B. LB simulations}

The LB simulations were carried out using the D3Q19 model with $24^{3}$ solvent nodes. The walls for the ions are placed as in the DPD simulations at $z_{\mathrm{wall}}=(0,10) \sigma$. Since the zero-velocity surface in a LB simulation is located, at first order in viscosity, halfway in between two rows of nodes, a shift has been added to the WCA wall potential in order to match the position of the zero-potential and zero-velocity planes. The grid spacing of the LB fluid is $a=0.5 \sigma$. The coupling constant of the fluid with the ions is $\zeta$ $=1.98 \sigma^{-1}(m \epsilon)^{1 / 2}$ which was derived by the mapping scheme proposed in Sec. V. The integration timestep for the ions as well as for the fluid was $\delta t=\tau=0.01 \sigma(m / \epsilon)^{1 / 2}$, bounce-back boundary conditions were applied on the fluid at the wall positions to create no-slip boundary conditions.

\section{RESULTS}

\section{A. Computational cost}

An important criterion when choosing a mesoscopic simulation method is its computational cost and efficiency. We have measured the time to calculate a single time step in both methods on an Athlon $\odot$ MP2200+CPU. The values are presented in Table II. The first two columns show the time spent on an uncharged system with 4320 solvent particles (DPD) with tunable-slip boundary conditions or 1728 solvent nodes (LB) in the above mentioned microgeometry with bounce-back boundary conditions. The LB method is nine times faster than the DPD method. We should note, however, that these values strongly depend on the density of solvent particles or the number of solvent nodes, respectively. The last two columns show the corresponding values for a charged system with the P3M in combination with the ELC algorithm for discretely charged walls. It is evident that most of the time is spent on the calculation of the electrostatic interactions for 60 ions and 60 counterions $\left(\sigma_{s}=0.208 \sigma^{-2}\right)$. If electrostatic interactions are considered, both methods are comparable with respect to their computational cost and efficiency, although it has to be noticed that the efficiency of P3M strongly depends on the chosen parameter values (Ewald parameter $\alpha=2.1875$, mesh size $32^{3}$ ). Depending on the choice of parameter and of system size the computational load of computing electrostatic calculation can be even lower than that of hydrodynamics.

\section{B. Fluid properties}

We first consider the dynamic properties of the bulk fluid. To measure the dynamic viscosity of the DPD fluid, which is needed for the parameter mapping, we fitted a plane Poiseuille flow as in Ref. 25. This procedure yields the value

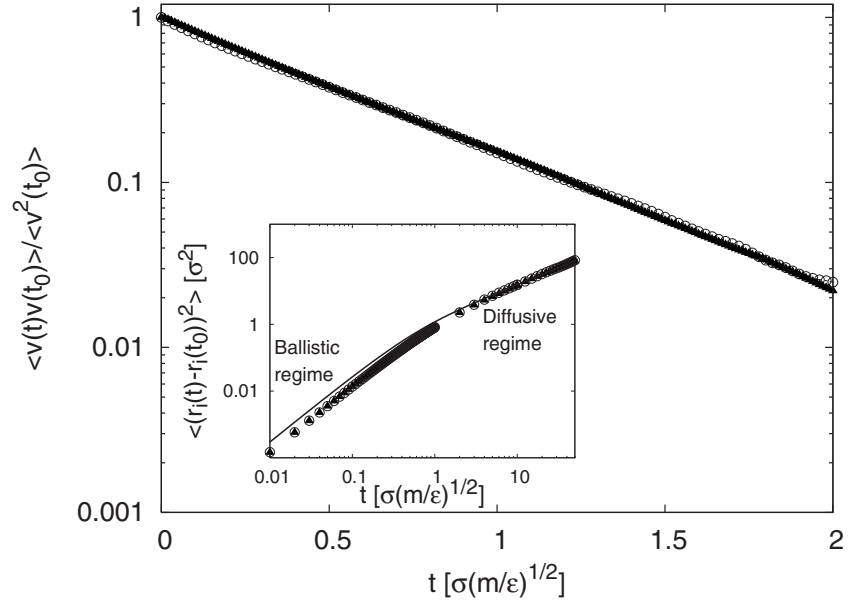

FIG. 1. Normalized velocity autocorrelation function for a DPD-fluid particle (open circles) with number density $\rho=3.75 \sigma^{-3}$, DPD friction coefficient $\gamma=5.0 \sigma^{-1}(m \epsilon)^{1 / 2}$, and for a LB/MD-fluid particle (filled triangles) with the same density and coupling constant $\zeta=1.98 \sigma^{-1}(m \epsilon)^{1 / 2}$. The characteristic decay time for the DPD method is $\tau_{\mathrm{DPD}}=(0.5162 \pm 0.0008) \sigma(\mathrm{m} / \epsilon)^{1 / 2}$ and for the LB method $\tau_{\mathrm{LB}}=(0.5218 \pm 0.0006) \sigma(m / \epsilon)^{1 / 2}$. Inset: Mean square displacement for a fluid DPD particle (open circles) and for a coupled LB/MD particle (filled triangles) compared to Eq. (24) (solid line).

$\eta=(1.334 \pm 0.003) \sigma^{-2}(m \epsilon)^{1 / 2}$ both for uncharged and charged fluids. Next we calculated the effective diffusion coefficient $D$ for a single tracer particle in the DPD method and matched it to the tracer diffusion in the LB model. The selfdiffusion coefficient $D$ can be obtained by exploiting a Green-Kubo expression, ${ }^{34}$ which relates $D$ to the velocity autocorrelation function (see Fig. 1),

$$
D=\frac{1}{3} \int_{t_{0}}^{\infty} d t\left\langle\mathbf{v}(t) \mathbf{v}\left(t_{0}\right)\right\rangle .
$$

Figure 1 shows that the velocity autocorrelation function decays exponentially over at least two orders of magnitude. Approximating the integrand in Eq. (22) by this exponential law we obtain $D_{\mathrm{DPD}}=(0.2581 \pm 0.0004) \sigma(m / \epsilon)^{-1 / 2}$ for the particle in the DPD fluid (at late times, one expects to see an algebraic long-time tail in the velocity autocorrelation function, but its contribution to $D$ is small and well within the error). A coinciding value of the self-diffusion coefficient in the LB/MD method can be obtained by setting the coupling constant to $\zeta=1.98 \sigma^{-1}(m / \epsilon)^{1 / 2}$. The two corresponding velocity autocorrelation functions then closely match each other (Fig. 1) and the resulting self-diffusion coefficient for LB/MD-fluid particles is given by $D_{\mathrm{LB}}$ $=(0.2609 \pm 0.0003) \sigma(m / \epsilon)^{-1 / 2}$.

Alternatively, the self-diffusion coefficient can be determined directly from the mean-square displacement of a single solvent particle at late times,

$$
D=\lim _{t \rightarrow \infty} \frac{\left\langle\left(\mathbf{r}_{i}(t)-\mathbf{r}_{i}\left(t_{0}\right)\right)^{2}\right\rangle}{6 t} .
$$

The results for the mean-square displacement are shown in the inset of Fig. 1. In agreement with standard theories, ${ }^{45,46}$ ballistic behavior $\left(\sim t^{2}\right)$ is observed at short times $\left(t \leq 0.75 \sigma(m / \epsilon)^{1 / 2}\right)$, but diffusive behavior dominates after a characteristic time which is roughly $t \geq 10 \sigma(m / \epsilon)^{1 / 2}$ for our 
TABLE III. Slip lengths for different layer friction coefficients $\gamma_{L}$, determined for uncharged fluid flow. The numerical results are compared to the theoretical prediction of Ref. 25.

\begin{tabular}{lcccc}
\hline \hline$\gamma_{L}\left[\sigma^{-1}(m \epsilon)^{1 / 2}\right]$ & 0.96 & 1.4049 & 3.1 & 6.1 \\
\hline$\delta_{B}$ (measured) $[\sigma]$ & $1.399 \pm 0.385$ & $0.782 \pm 0.246$ & $0.248 \pm 0.231$ & $0.000 \pm 0.197$ \\
$\delta_{B}$ (theory) $[\sigma]$ & 1.249 & 0.780 & 0.226 & 0.000 \\
\hline \hline
\end{tabular}

model parameters. A linear regression of the diffusive regime yields $D_{\mathrm{DPD}}=(0.2698 \pm 0.0002) \sigma(m / \epsilon)^{-1 / 2}$ and $D_{\mathrm{LB}}$ $=(0.2617 \pm 0.0005) \sigma(m / \epsilon)^{-1 / 2}$, which is in rough agreement with the Green-Kubo results. It is remarkable that the numerical results obtained with both methods lie almost on top of each other. Assuming that the velocity of a single particle propagates according to an Ornstein-Uhlenbeck process, the full-time mean-square displacement can be calculated analytically as a function of the effective friction coefficient $\zeta_{e}$ $=k_{B} T / D$ and is given by ${ }^{45}$

$$
\begin{aligned}
\left\langle\left(\mathbf{r}_{i}(t)-\mathbf{r}_{i}\left(t_{0}\right)\right)^{2}\right\rangle= & 6 \frac{k_{B} T}{m \zeta_{e}} t+\frac{\left\langle\mathbf{v}^{2}\left(t_{0}\right)\right\rangle}{\zeta_{e}^{2}}\left(1-e^{-\zeta_{e} t}\right)^{2} \\
& -\frac{k_{B} T}{m \zeta_{e}^{2}}\left(3-4 e^{-\zeta_{e} t}+e^{-2 \zeta_{e} t}\right) .
\end{aligned}
$$

This prediction is shown as the straight line in the inset of Fig. 1. It is in reasonable agreement with the numerical results.

Finally, we consider uncharged DPD fluids in slit geometry and establish the values of the slip length $\delta_{B}$ and the hydrodynamic boundary positions $z_{B}$ for different values of the surface friction parameter $\gamma_{L}$. By a combination of plane Poiseuille flow and plane Couette flow simulations, it is possible to determine $\delta_{B}$ and $z_{B}$ independently. ${ }^{25}$ The resulting hydrodynamic boundary positions were found to be located at $\left|z_{B}\right|=(3.866 \pm 0.265) \sigma$, and the corresponding results of the slip length compared to the theoretical prediction of the analytical expression derived in Ref. 25 are shown in Table III.

Having matched the model parameters in the DPD and the LB/MD method, we can now proceed to simulate the counterion-induced EOF and to compare the results.

\section{EOF: Weak coupling regime}

First we consider the counterion distribution in the channel with inhomogeneously charged walls in the weak coupling regime $(\Xi=1.31)$. DPD simulations were carried out for ten different external electric field strengths between $E_{x}$ $=0.1-1.0 k_{B} T / e \sigma$. Figure 2 shows that the counterion distribution between the walls is not perturbed by the applied fields nor the discreteness of charges in the walls in the accessible $z$-range. For all fields, it is in very good agreement with the theoretical prediction of the Poisson-Boltzmann equation (7) with the only fit parameter $\rho_{0}$ $=(0.0176 \pm 0.0001) \sigma^{-3}$. The inset of Fig. 2 compares the counterion distribution in the DPD fluid with the corresponding ion distribution in the LB/MD fluid for a field strength $E_{x}=1.0 k_{B} T / e \sigma$. The simulation results are nearly identical and follow again closely the prediction of the electrostatic theory. In addition, we also measured the electric field $E(z)$ by a test charge method. The results are shown in Fig. 3, they also agree very well with the prediction of the PoissonBoltzmann theory, Eq. (8).

The influence of the partial-slip boundary conditions is presented in Fig. 4 for varying field strength $E_{x}$. In agreement with the theoretical prediction, Eq. (9), we find that $v_{x} \propto E_{x}$ for all slip lengths. Varying the slip length has a quite dramatic effect on the magnitude of the flow profiles. All numerical results are in reasonable agreement with Eq. (9), especially if one bears in mind that the theoretical curves have an uncertainty due to the numerical error of $\delta_{B}$.

The comparison of the DPD and the LB/MD flow profiles is finally presented in Fig. 5 for no-slip boundary conditions. Here we have shifted the effective channel width in the DPD method from $8 \sigma$ to $8.28 \sigma$, such that the hydrodynamic boundaries were now located at $\left|z_{B}\right|=4.030 \pm 0.357 \sigma$ like in the LB/MD fluid. Figure 5 shows that the flow profiles obtained with both methods are then identical and agree well with the theoretical prediction, Eq. (9), evaluated with $\delta_{B}=0$ and $\left|z_{B}\right|=4 \sigma$.

\section{EOF: Homogeneously and inhomogeneously charged walls}

The results shown up to now have been obtained in system where discrete charges were placed randomly in the walls. For comparison, we have also studied systems with homogeneously charged walls with DPD simulations. Sur-

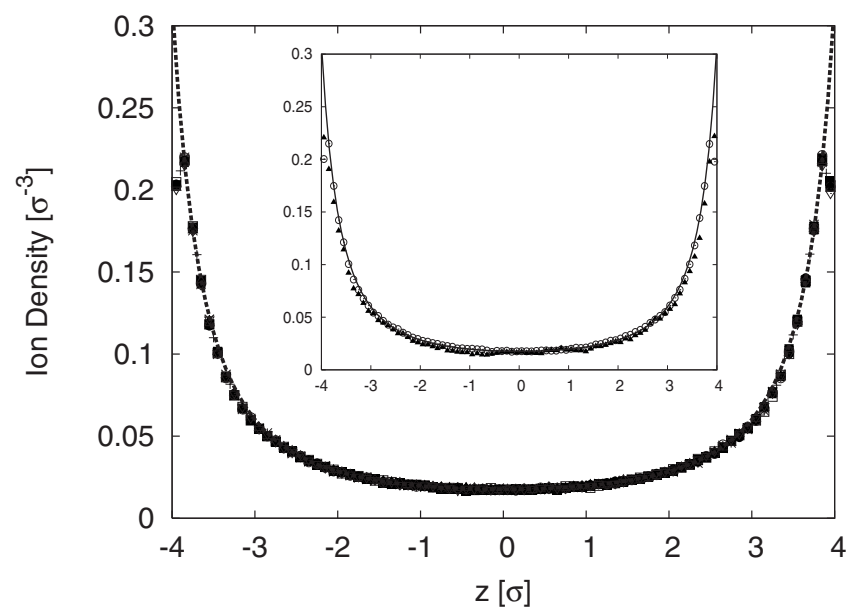

FIG. 2. Counterion density distribution for the DPD fluid in the weak coupling limit $(\Xi=1.31)$ between two charged walls for external electric field strengths between $E_{x}=0.1-1.0 k_{B} T / e \sigma$. The external perpendicular electric field does not perturb the ion density distribution. The dashed line presents the theoretical prediction of the PB theory for an ion density in the middle of the channel of $\rho_{0}=(0.0176 \pm 0.0001) \sigma^{-3}$. Inset: Comparison of counterion distribution for the DPD method (circles) and for the LB method (triangles) with a field strength $E_{x}=1.0 k_{B} T / e \sigma$. The straight line is again the theoretical prediction of the Poisson-Boltzmann theory. 


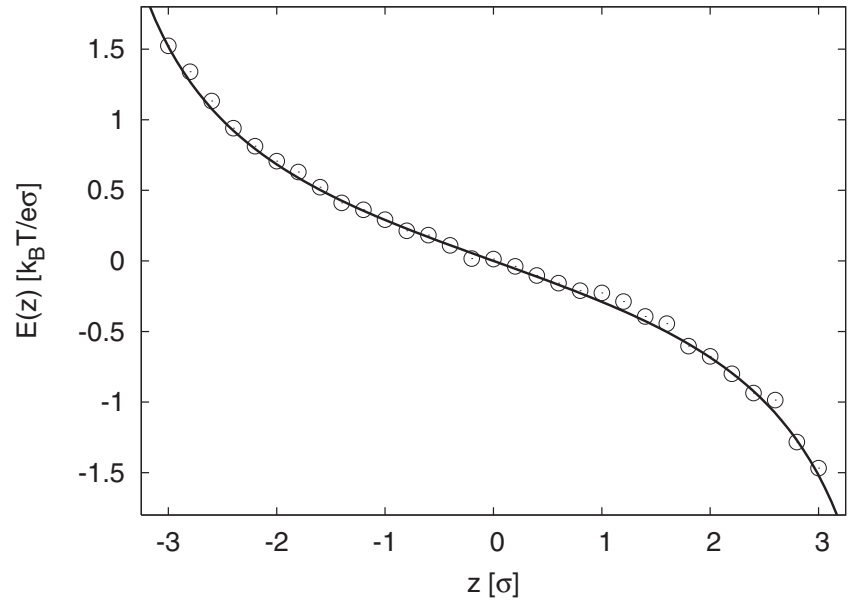

FIG. 3. Electric field $E(z)$ in the solvent measured by a test charge method in the weak coupling limit $(\Xi=1.31)$. The dashed line shows the theoretical prediction of Eq. (8).

face charge inhomogeneities may lead to electrofriction and slow down the fluid at the wall. ${ }^{47}$ Since the electrostatic potential between two equally and homogeneously charged walls is constant, the charges on the wall can be omitted and it suffices to study a fluid with ions confined between uncharged walls. The simulations were carried out using the MMM2D-algorithm ${ }^{42,43}$ and the parameters given in Table III for the weak coupling (Poisson-Boltzmann) regime. The ion density $\rho$ was $0.0525 \sigma^{-3}$ in all simulations. Figure 6 presents the numerical results for various slip lengths for homogeneously and inhomogeneously charged walls, compared to the theory of Eq. (9) for an external field of $E_{x}$ $=1.0 k_{B} T / e \sigma$. All flow profiles for homogeneously, respectively, inhomogeneously charged walls are identical. These results are consistent with theories ${ }^{47,48}$ - the electrostatic interaction is moderate and drastic deviations have only been reported for strongly interacting systems. ${ }^{47}$ In the weak coupling regime, the influence of electrofriction on the flow profiles both for no-slip and partial-slip boundaries is thus negligible.

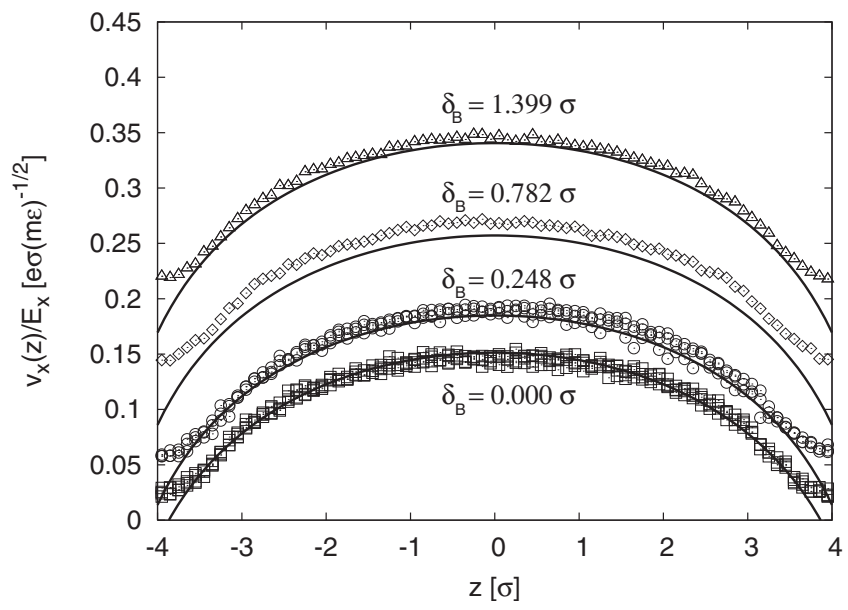

FIG. 4. Flow profiles for the DPD method with various field strengths $E_{x}$ $=0.8-1.0 k_{B} T / e \sigma$ for varying slip lengths in the weak coupling regime ( $\Xi$ $=1.31$ ). The hydrodynamic boundary positions for the DPD method are at $\left|z_{B}\right|=(3.866 \pm 0.265) \sigma$. The straight lines represent the theoretical prediction of Eq. (9).

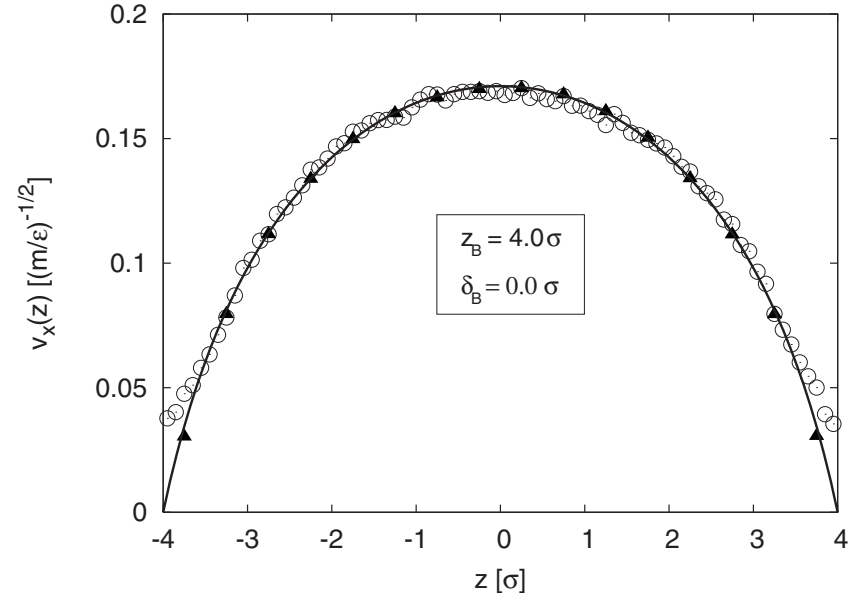

FIG. 5. Flow profile for the DPD (circles) and the LB/MD method (triangles) with no-slip boundary conditions. The straight line is the theoretical prediction of Eq. (9) with $\left|z_{B}\right|=4.0 \sigma$ and $\delta_{B}=0.0 \sigma$.

\section{E. EOF: Intermediate coupling regime}

Finally, we consider a situation where no analytical theory is available, and study the intermediate coupling regime with $\Xi=4.19$. The simulation results for the counterion density distribution are presented in Fig. 7. The distribution can neither be fully described by the strong coupling theory [Eq. (10)], shown as straight line, nor by the PoissonBoltzmann theory [Eq. (7)], shown as dashed line. The inset of Fig. 7 shows that the counterion distribution close to the walls decays exponentially with the Gouy-Chapman length $\mu$, indicating the presence of a layer of highly adsorbed counterions. This corresponds to the behavior predicted by the strong coupling theory [Eq. (10)]. In the bulk, however, the data are better described by the Poisson-Boltzmann theory in agreement with Ref. 24. Thus, the intermediate regime bears characteristics of both the strong coupling and the Poisson-Boltzmann regime. For the former regime, a recently developed method ${ }^{49}$ might turn out to be useful. These

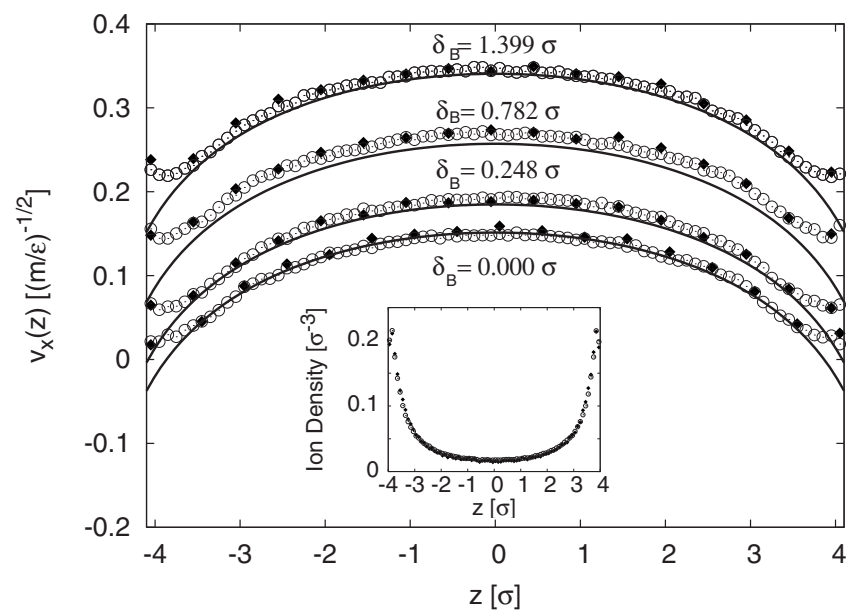

FIG. 6. Flow profile for various slip lengths with homogeneously (filled diamonds) and inhomogeneously charged walls (circles) for $E_{x}$ $=1.0 k_{B} T / e \sigma$ in comparison to the theoretical prediction of Eq. (4). Inset: Counterion distribution with $\sigma_{A}=0.208 e \sigma^{-2}$ between homogeneously, respectively, inhomogeneously charged walls. Only slight deviations can be observed. 


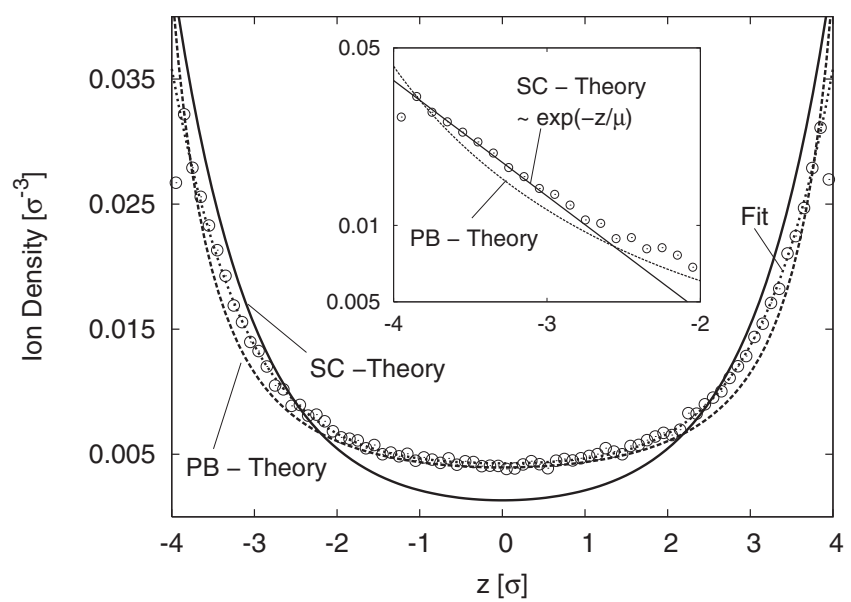

FIG. 7. Counterion density distribution with $\rho=0.0104 \sigma^{-3}$ in the intermediate coupling regime with $\Xi=4.19$ for a surface ion density $\sigma_{s}=0.042 \sigma^{-2}$. The straight line shows the theoretical prediction of the strong coupling limit [Eq. (10)]. Neither the strong coupling theory (straight line) nor the Poisson-Boltzmann theory (dashed line at the bottom) is able to reproduce the results. The fit function is represented by the dotted line. Inset: Blowup of the $z$-range between $-4.0 \sigma$ and $-2.0 \sigma$ in a logarithmical plot. The counterion density distribution decays exponentially proportional to the GouyChapman length of $\mu=0.955 \sigma$.

results are consistent with Refs. 24 and 50, where it was shown that in case of a single charged plate, neither the Poisson-Boltzmann nor the strong coupling theory are applicable at distances from the plate $d^{\prime}$ in the range $\sqrt{\Xi}$ $<d^{\prime} / \mu<\Xi$. Inserting the parameters of $\Xi$ and $\mu$, this corresponds to the $z$-range $|z|>2 \sigma$.

Although we have no analytical theory, we can still fit the counterion density with a purely heuristic function whose functional form is inspired by the prediction of the strong coupling theory, Eq. (10),

$$
\rho^{t}(z)=\rho_{(1)}^{t}\left(e^{-(z-d / 2) / \mu}+e^{(z+d / 2) / \mu}\right)+f_{\text {corr }}(z),
$$

where $\rho_{(1)}^{t}$ is a fitting parameter and $f_{\text {corr }}(z)$ a phenomenological correction function. A good fit can be obtained with $f_{\text {corr }}(z)=\rho_{(2)}^{t} \cos (\phi z)$ with $\phi$ and $\rho_{(2)}^{t}$ being two additional fit parameters. The result of the fit to this expression [with fitting parameters values $\rho_{(1)}^{t}=(8.31 \pm 0.10) \times 10^{-6} \sigma^{-3}, \quad \rho_{(2)}^{t}$ $=(3.18 \pm 0.05) \times 10^{-3} \sigma^{-3}$, and $\phi=(0.416 \pm 0.022) \sigma^{-1}$, respectively] is presented as a dotted line in Fig. 7. Combining Eq. (25) with the Stokes equation, Eq. (3), and integrating it using Eq. (4) as boundary condition, allows to reproduce the EOF profiles very nicely (Fig. 8). Our result demonstrates that the Stokes equation is still applicable in the intermediate coupling regime. Furthermore, Fig. 8 shows that we still do not observe a noticeable effect of the surface charge inhomogeneity, compared to simulations with homogeneously charged walls. Thus, electrofriction plays a negligible role in the intermediate as well as in the weak coupling regime and seems to be a prominent effect only in the strong coupling regime.

\section{SUMMARY}

We have carried out two types of mesoscopic simulations, namely, DPD and coupled LB/MD, of the counterion-

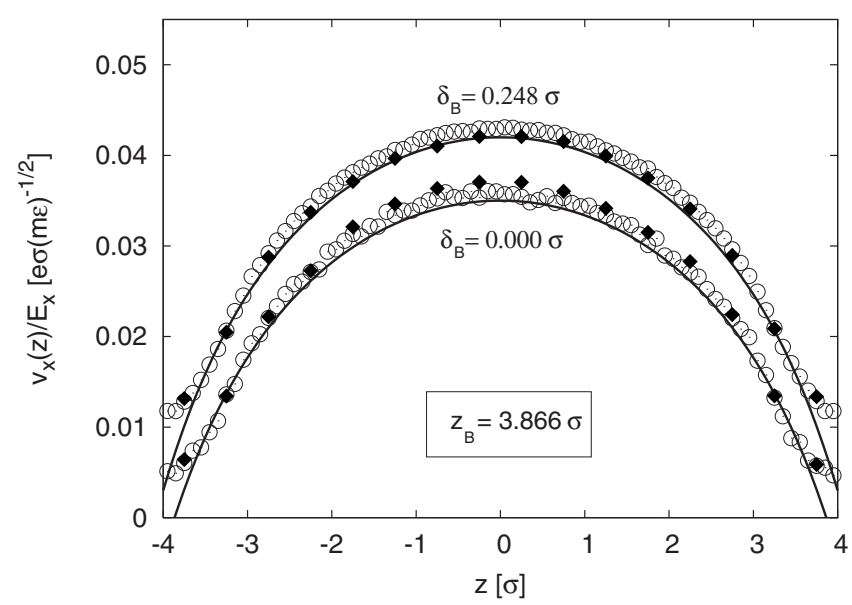

FIG. 8. Counterion-induced EOF for the intermediate regime $(\Xi=4.19)$ with $\rho=0.0104 \sigma^{-3}, E_{x}=10.0 k_{B} T / e \sigma$, and surface ion density $\sigma_{s}=0.042 \sigma^{-2}$ with $\gamma_{L}=6.1 \sigma^{-1}(m \epsilon)^{1 / 2}\left(\delta_{B}=(0.000 \pm 0.197) \sigma\right)$ and $\gamma_{L}=6.1 \sigma^{-1}(m \epsilon)^{1 / 2}\left(\delta_{B}\right.$ $=(0.248 \pm 0.231) \sigma)$ for homogeneously (filled diamonds) and inhomogeneously charged walls (circles). The straight lines show the calculated flow profile for $\left|z_{B}\right|=3.866 \sigma$ and for slip lengths as indicated.

induced EOF in slit channels. We have proposed a mapping scheme that allows to match quantitatively the parameters of the two models. The cost of calculation time for electrostatic problems in both methods turned out to be nearly identical.

We have considered different electrostatic coupling regimes. In the weak coupling regime, the numerical results for the ion density and the electric field are in good agreement with the predictions of the Poisson-Boltzmann theory. We have derived analytical expressions for the corresponding EOF profile in presence of no-slip as well as partial-slip boundary conditions, which also agree well with the simulations. In addition, we have considered the intermediate coupling regime where no analytical theory is available. A heuristic function was used to fit the counterion distribution in this transient regime. The Stokes equation can still be used to calculate the corresponding EOF profiles, with very good results as compared to the simulations.

In all systems under consideration, electrofriction effects were found to be negligible. In contrast, the presence of partial slip changes the magnitude of the flow profiles drastically even if the slip lengths are very small. ${ }^{3}$ This may facilitate the generation of flow profiles in microfluidic applications.

\section{ACKNOWLEDGMENTS}

We thank B. Dünweg for enlightening discussions. This work was funded by the Volkswagen Stiftung (Grant No. I/80431). The simulations were carried out at the John-von Neumann Supercomputing Center in Jülich (NIC), the High Performance Computing Center Stuttgart (HLRS), and the High Performance computing cluster PC2 of the University of Paderborn.

${ }^{1}$ D. J. Harrison, K. Fluri, K. Seiler, Z. H. Fan, C. S. Effenhauser, and A. Manz, Science 261, 895 (1993).

${ }^{2}$ D. R. Reyes, D. Iossifidis, P. A. Auroux and A. Manz, Anal. Chem. 74, 2623 (2002).

${ }^{3}$ L. Bocquet and J.-L. Barrat, Soft Matter 3, 685 (2007). 
${ }^{4}$ J. Israelachvili, Intermolecular and Surface Forces (Academic, London, 1991).

${ }^{5}$ R. J. Hunter, Foundations of Colloid Science (Clarendon, Oxford, 1987).

${ }^{6}$ J.-L. Viovy, Rev. Mod. Phys. 72, 813 (2000).

${ }^{7}$ U. Frisch, B. Hasslacher, and Y. Pomeau, Phys. Rev. Lett. 56, 1505 (1986).

${ }^{8}$ G. R. McNamara and G. Zanetti, Phys. Rev. Lett. 61, 2332 (1988).

${ }^{9}$ R. Benzi, S. Succi, and M. Vergassola, Phys. Rep. 222, 145 (1992).

${ }^{10}$ S. Chen and G. D. Doolen, Annu. Rev. Fluid Mech. 30, 329 (1998).

${ }^{11}$ P. J. Hoogerbrugge and J. M. Koelman, Europhys. Lett. 19, 155 (1992).

${ }^{12}$ P. Español and P. B. Warren, Europhys. Lett. 30, 191 (1995).

${ }^{13}$ R. D. Groot and P. B. Warren, J. Chem. Phys. 107, 4423 (1997).

${ }^{14}$ A. Malevanets and R. Kapral, J. Chem. Phys. 110, 8605 (1999).

${ }^{15}$ A. Malevanets and R. Kapral, J. Chem. Phys. 112, 7260 (2000).

${ }^{16}$ P. B. Warren, Int. J. Mod. Phys. C 8, 889 (1997).

${ }^{17}$ F. Capuani, I. Pagonabarraga, and D. Frenkel, J. Chem. Phys. 121, 973 (2004).

${ }^{18}$ D. Frenkel and B. Smit, Understanding Molecular Simulation (Academic, Orlando, 2001).

${ }^{19}$ R. R. Netz and H. Orland, Eur. Phys. J. E 1, 67 (2000).

${ }^{20}$ A. G. Moreira and R. R. Netz, Phys. Rev. Lett. 87, 078301 (2001).

${ }^{21}$ A. G. Moreira and R. R. Netz, Europhys. Lett. 52, 705 (2000).

${ }^{22}$ M. Gouy, J. Phys. Theor. Appl. 9, 457 (1910).

${ }^{23}$ D. L. Chapman, Philos. Mag. 25, 475 (1913).

${ }^{24}$ H. Boroudjerdi, Y.-W. Kim, A. Naji, R. R. Netz, X. Schlagberger, and A. Serr, Phys. Rep. 416, 129 (2005).

${ }^{25}$ J. Smiatek, M. P. Allen, and F. Schmid, Eur. Phys. J. E 26, 115 (2008).

${ }^{26}$ R. Pit, H. Hervet, and L. Leger, Phys. Rev. Lett. 85, 980 (2000).

${ }^{27}$ D. Tretheway and C. Meinhart, Phys. Fluids 14, L9 (2002).

${ }^{28}$ C. Neto, D. R. Evans, E. Bonaccurso, H.-J. Butt, and V. S. J. Craig, Rep. Prog. Phys. 68, 2859 (2005).

${ }^{29}$ S. Succi, The Lattice Boltzmann Equation for Fluid Dynamics and Beyond (Oxford University Press, New York, 2001).
${ }^{30}$ B. Dünweg, U. D. Schiller, and A. J. C. Ladd, Phys. Rev. E 76, 036704 (2007).

${ }^{31}$ P. Ahlrichs and B. Dünweg, Int. J. Mod. Phys. C 9, 1429 (1998).

${ }^{32}$ M. Revenga, I. Zuniga, and P. Español, Comput. Phys. Commun. 121122, 309 (1999)

${ }^{33}$ C. Marsh, E. Backx, and M. H. Ernst, Phys. Rev. E 56, 1676 (1997).

${ }^{34}$ M. P. Allen and D. J. Tildesley, Computer Simulations of Liquids (Oxford Science, Oxford, 1987).

${ }^{35}$ J. A. Backer, C. P. Lowe, H. C. J. Hoefsloot, and P. D. Iedema, J. Chem. Phys. 122, 154503 (2005).

${ }^{36}$ ESPRESSO, Homepage http://www.espresso.mpg.de.

${ }^{37}$ A. Arnold, B. A. Mann, H. -J. Limbach and C. Holm, GWDG-Bericht: Forschung und wissenschaftliches Rechnen 2003 63, Gesellschaft für wissenschaftliche Datenverarbeitung mbh, Göttingen (2003).

${ }^{38}$ H. J. Limbach, A. Arnold, B. A. Mann, and C. Holm, Comput. Phys. Commun. 174, 704 (2006).

${ }^{39}$ R. W. Hockney and J. W. Eastwood, Computer Simulation Using Particles (McGraw-Hill, New York, 1981).

${ }^{40}$ A. Arnold, J. de Joannis, and C. Holm, J. Chem. Phys. 117, 2496 (2002).

${ }^{41}$ M. Deserno and C. Holm, J. Chem. Phys. 109, 7678 (1998).

${ }^{42}$ A. Arnold and C. Holm, Comput. Phys. Commun. 148, 327 (2002).

${ }^{43}$ A. Arnold and C. Holm, Chem. Phys. Lett. 354, 324 (2002).

${ }^{44}$ J. D. Weeks, D. Chandler, and H. C. Andersen, J. Chem. Phys. 54, 5237 (1971).

${ }^{45}$ W. Paul and J. Baschnagel, Stochastic Processes: From Physics to Finance (Springer, Berlin, 1999).

${ }^{46}$ N. G. van Kampen, Stochastic Processes in Physics and Chemistry (NH Elsevier, Amsterdam, 2003).

${ }^{47}$ R. R. Netz, Phys. Rev. Lett. 91, 138101 (2003).

${ }^{48}$ L. Joly, C. Ybert, E. Trizac, and L. Bocquet, Phys. Rev. Lett. 93, 257805 (2004).

${ }^{49}$ M. M. Hatlo and L. Lue, Soft Matter 5, 125 (2009).

${ }^{50}$ Y. Burak, A. Andelman, and H. Orland, Phys. Rev. E 70, 016102 (2004). 